\title{
Recent Advances in Microstructured Fibers for Power Delivery
}

\author{
David Richardson, Marco Petrovich, John Hayes, \\ Franceso Poletti, Sonali Dasgupta, Xian Feng, Wei Loh and Neil Broderick \\ Optoelectronics Research Centre, University of Southampton, Southampton, SO17 1BJ, United Kingdom \\ djr@orc.soton.ac.uk
}

\begin{abstract}
We report recent advances in the development of fibers for the delivery of both single and heavily multimode laser beams in spectral regimes spanning the visible to mid-IR.

(C) 2009 Optical Society of America

OCIS codes: (060.2280) Fiber design and fabrication; (060.2400) Fiber properties; (060.4005) Microstructured fibers
\end{abstract}

\section{Introduction}

Microstructured optical fiber (MOF) technology provides a powerful means to develop fibers with unique and enabling properties with potential uses spanning a very wide range of applications. Of all the opportunities provided by MOF technology amongst the most novel and intriguing are: (a) the possibility to guide light in air (through photonic band-gap or low density of state effects); (b) single mode guidance over extended spectral ranges (referred to as endlessly single mode (ESM) guidance); and (c) the fabrication of fibers with very much larger values of Numerical Aperture (NA) than possible using conventional fiber fabrication approaches. It is also important to realize that the MOF technology allows the fabrication of fibers using a single material - considerably enhancing the ease of producing fibers in new materials, such as polymers and compound glasses. This offers access to material properties that are substantially different to those of silica based glasses allowing, for example, fibers with transmission extending into the mid-IR, or ultrahigh values of nonlinearity per unit length. In the following sections we review recent progress in the design and fabrication of fibers that exploit several of these opportunities for the delivery of high power laser radiation and/or broadband light in different spectral regimes.

\section{Photonic band gap fibers:}

The primary benefits of photonic band gap fibers for power delivery relate to the low nonlinearity associated with the air-core guidance and the prospects for reduced losses in wavelength regimes in which the inherent glass loss would make solid-core variants unusable. Minimizing the modal overlap with the solid regions of the fiber is critical to optimizing the performance of such fibers from these perspectives. Moreover, in many applications, particularly those involving short pulses and/or relatively short delivery lengths, maintaining single transverse mode guidance is a key issue, as is maintaining the maximum usable bandwidth. With this in mind we have conducted extensive numerical and theoretical studies to identify fiber designs providing robust, broadband guidance [1-3]. In Figure 1
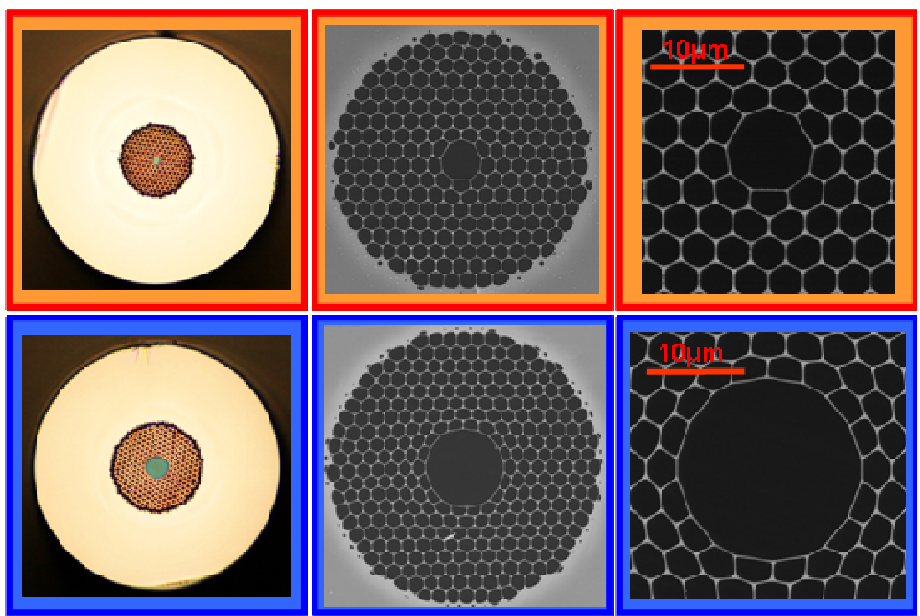

Figure $13 \mathrm{c}$ (Top) single mode PBG fiber incorporating a 3-cell core; (Bottom) 19we show two fibers fabricated in our facilities cell PBGF for extended near-IR broadband transmission

with a suitable choice of geometry according to the design rules derived. These pictures show fibers with two sizes of defect created by the omission of 3 and 19 central capillaries. Measurements confirm the broad usable bandwidths and show that the effective modal overlap with the solid regions of the fiber decreases steadily with increasing core size as predicted [4]. It is however to be appreciated that this is associated with a corresponding increase in the number of modes supported by the structure. Only by going to the smallest scale core is robust single mode 
guidance achievable, as reported in [4]. Exploiting the low overlap factors associated with 19-cell designs we have developed fibers operating at wavelengths $>2.5 \mu \mathrm{m}$, well beyond the accepted transparency window of silica [2].

\section{Large mode area - endlessly single mode fibers}

Endlessly single mode (ESM) fibers are of interest for a range of potential applications and hexagonally stacked fibers with differing core structure have been developed and characterized in terms of their modedness, effective area, and bend-loss characteristics. In many single wavelength applications conventional doped fiber technology can arguably deliver fibers capable of similar performance. However, MOF technology offers potential advantage, when single mode guidance is required over extended wavelength ranges. For example, we have recently developed structures capable of delivering both fundamental and second harmonic radiation from a pulsed Q-switched $\mathrm{Nd}$ :YAG laser as required for various direct-write materials processing applications [5]. In doing this work we identified additional parasitic modes guided due to bandgap/low density of states effects capable of degrading the mode quality and which may limit the utility of such fibers in certain instances [6]. Finally, it is to be appreciated that the use of ESM fibers in additional wavelength regimes has also become the focus of considerable attention with the first reports of such fibers being made in new materials such as Tellurite

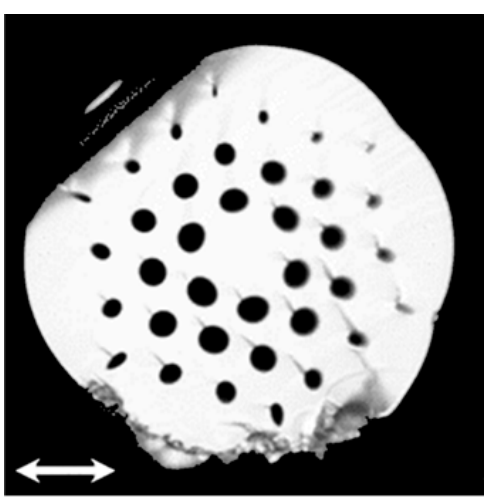
$100 \mu \mathrm{m}$

Figure 2 LMA Tellurite fiber with an effective area of $\sim 2000 \mu \mathrm{m}^{2}$ glass (transparent at wavelengths up to $5 \mu \mathrm{m}$ ) [7] and in silver halides (capable of guiding light at wavelength beyond $20 \mu \mathrm{m}$ ) [8]. As well as being of interest for high power laser delivery, such fibers are of use in applications such as modal filtering in space-borne astronomy [9], and broadband mid-IR supercontinuum generation [7].

\section{High NA JAC fibers}

High-NA, large core Jacketed Air Clad (JAC) fibers are of relevance to a range of applications most notably in the context of fiber lasers and multimode laser diode beam delivery since they allow confinement of the guided light to a far smaller cross-sectional area than possible in either allglass, or polymer-clad structures. This tighter optical confinement can be used to make shorter fiber amplifier and laser device, reducing the impact of fiber nonlinearities and/or dispersion. Numerical apertures approaching unity are now possible. In addition, the technology also allows for the ready implementation of fibers with different cross-sectional profiles. For example, in Figure 3 we show an SEM of a square JAC fiber recently developed at Southampton for high power laser beam delivery in which the square near field profile is used to provide benefit in terms of pixel-definition in

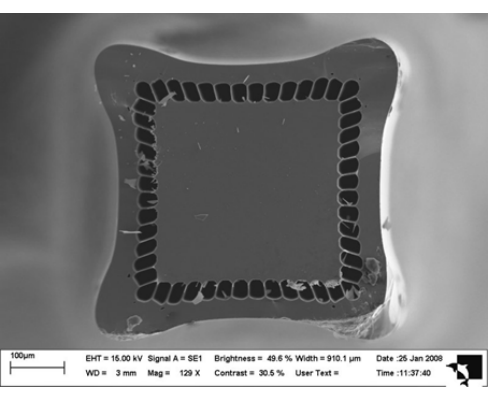

Figure 3 Large core square JAC fiber for high power laser delivery. applications such as flat screen display manufacture [10], amongst others.

\section{Acknowledgements}

This work was funded in part by UK Technology Strategy Board grants LAMPS and PFIDEL and UK EPSRC grant $\mathrm{EP} / \mathrm{C} 515668 / 1$.

\section{References}

[1] R.Amezcua-Correa et al., Optics Express 2006 Vol.14(17) pp.7974-7985

[2] R.Amezcua-Correa et al., ECOC 2006 Cannes 24-28 Sep 2006

[3] R.Amezcua-Correa et al., Optics Express 2008 Vol.16(2) pp.1142-1149

[4] M.N.Petrovich, F.Poletti, A.van Brakel, D.J.Richardson, Optics Express 2008 Vol.16(6) pp.4337-4346

[5] T.Delmonte et al., SPIE Conference: Optics/Photonics in Security Stockholm 11-14 Sep 2006 6400-04

[6] J.C.Flanagan et al., Optics Express 2008 Vol.16(23) pp.18631-18645

[7] X.Feng et al., Optics Express 2008 Vol.16(18) pp.13651-13655

[8] A. Millo, L. Lobachinsky, A. Katzir, IEEE Photonics Technology Letters 2008, Vol.20,pp 869-871.

[9] J.C.Flanagan, D.J.Richardson, M.J.Foster, I.Bakalski, Optics Express 2006 Vol.14(24) pp.11773-11786

[10] J.R.Hayes et al., Optics Express 2006 Vol.14(22) pp.10345-10350 